\title{
Los manglares de Tabasco, una reserva natural de carbono
}

\author{
Elvia Moreno Cáliz ${ }^{1}$ \\ Armando Guerrero Peña ${ }^{2}$ \\ Ma. del Carmen Gutiérrez Castorena ${ }^{1}$ \\ Carlos Alberto Ortiz Solorio ${ }^{1}$ \\ David Jesús Palma López ${ }^{2}$
}

\begin{abstract}
RESUMEN
En el Panel Intergubernamental de las Naciones Unidas sobre el Cambio Climático, se ha indicado que el secuestro de carbono por las plantas es una alternativa de bajo costo para reducir el contenido de dióxido de carbono $\left(\mathrm{CO}_{2}\right)$ atmosférico. Por ello, el manejo forestal y de humedales representa una oportunidad para el almacenamiento de carbono. En el caso de los suelos orgánicos de manglares, los promedios de descomposición son bajos y su potencial de almacenamiento de carbono es alto, por lo que pueden ser una alternativa para el secuestro de carbono. En esta investigación se cuantificó la reserva de carbono en suelos orgánicos con vegetación de mangle (Rhizophora mangle L. y Laguncularia racemosa (L.) Gaertn) en la parte oeste del estado de Tabasco, México, encontrándose que el contenido de carbono fluctúa entre $47 \mathrm{~kg} \mathrm{C} \mathrm{m}^{-2}$ y $82 \mathrm{~kg} \mathrm{C} \mathrm{m}^{-2}$, lo cual refleja el gran potencial que tienen.
\end{abstract}

PALABRAS CLAVE:

Histosoles, humedales, manglares, suelos tropicales.

\begin{abstract}
The Intergovernmental Panel on Climate Change of the United Nations has indicated that carbon sequestration by plants is a low cost alternative to reduce atmospheric carbon dioxide $\left(\mathrm{CO}_{2}\right)$. Forest and wetlands management could be an opportunity for carbon storage. In organic soils of mangroves, the decomposition is low leading to a high potential capacity for carbon storage. This is a reason why this ecosystem could be an alternative the carbon sequestration. The carbon stock in mangrove's soils of Rhizophora mangle L. and Laguncularia racemosa (L.) Gaertn was quantified, in the west of Tabasco state, Mexico. The carbon storage capability was recorded to be between $47 \mathrm{~kg} \mathrm{C} \mathrm{m}^{-2}$ to $82 \mathrm{~kg} \mathrm{C} \mathrm{m}^{-2}$ which reflects the great potential of this type of soil.
\end{abstract}

KEY WORDS:

Histosols, wetlands, mangroves, tropical soils. 


\section{INTRODUCCIÓN}

A principios del año 2001, en el Panel Intergubernamental de Naciones Unidas sobre el Cambio Climático se presentó el tercer informe de evaluación sobre las tendencias actuales en el incremento de dióxido de carbono $\left(\mathrm{CO}_{2}\right)$ y se concluyó lo siguiente: la temperatura media de la superficie terrestre aumentará entre 1.4 y 5.8 grados centígrados en los próximos 100 años; el nivel de los océanos subirá entre 0.1 y 0.9 metros; habrá más sequía en zonas áridas y cambios sustanciales en los regímenes de precipitación. Se indicó además, que los mayores incrementos de $\mathrm{CO}_{2}$ atmosférico han ocurrido desde 1950, lo cual se atribuye principalmente a dos actividades humanas: cambios en la cobertura vegetal, por el uso del suelo y por el consumo de combustibles fósiles (Lal et al., 1998). Todo esto ha provocado un mayor efecto invernadero natural y generado un cambio climático no deseado; así mismo, el calentamiento del aire a nivel planetario ha originado el incremento del nivel del mar y efectos adversos sobre la agricultura y biodiversidad (Wallace, 2000). En este panel también se indicó que la captura y almacenamiento de las emisiones de $\mathrm{CO}_{2}$ por las plantas puede ser una de las alternativas de menor costo para reducir dichas emisiones.

El manejo de la vegetación forestal y de humedales puede representar una oportunidad para el almacenamiento significativo del carbono (C); por ejemplo, cuando un sistema agrícola es convertido a vegetación forestal con poco manejo, se puede acumular en promedio desde 0 megagramos de carbono por hectárea al

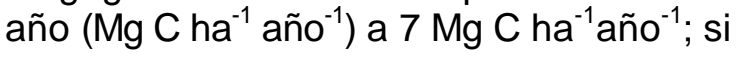
este cambio de uso del suelo se da en regiones templadas se alcanzaría de 0.2

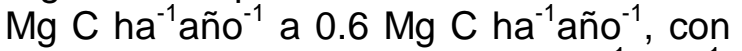
un promedio cercano a $0.3 \mathrm{Mg} \mathrm{C} \mathrm{ha-}^{-1}$ año $^{-1}$; mientras que en sistemas forestales tropicales y subtropicales este valor sería de $1.0 \mathrm{Mg} \mathrm{C}$ ha $^{-1}$ año- a $7.4 \mathrm{Mg} \mathrm{C} \mathrm{ha-1} a^{-1} \mathrm{ano}^{-1}$ con un promedio de $2.0 \mathrm{Mg} \mathrm{C} \mathrm{ha-}$ año- $^{-1}$ (Izaurralde et al., 2001).
Los manglares constituyen un importante recurso forestal en toda la banda intertropical del planeta; son los árboles que sostienen la biodiversidad entre los ecosistemas costeros tropicales, tienen un efecto sobre las áreas forestadas intermareales e incluso pueden influir tierra adentro. Los manglares constituyen una de las mejores opciones dentro de los humedales costeros, en la captura de carbono para reducir los efectos que induce la ruptura de la capa atmosférica de ozono y el efecto invernadero sobre el planeta. Estos cambios están afectando los patrones de temperatura, precipitación pluvial, depresiones tropicales, huracanes, variación del nivel medio del mar y descargas de ríos (Yáñez et al., 1998).

En el caso del suelo, éste también representa un papel importante en el ciclo del carbono (C) de la tierra porque en él se encuentra la principal reserva del ecosistema terrestre. Se ha estimado que el carbono orgánico (CO) en suelos del mundo es de 1500 pico gramos (pg), cerca de 2.1 veces más que en la atmósfera y cerca de 2.7 veces más que la reserva biótica que comprende a las plantas de la tierra (Neill et al., 1998). Esto significa que existe aproximadamente tres veces más carbono en los suelos que en la vegetación; más aún, se ha encontrado que el mayor promedio de carbono terrestre ocurre en los suelos orgánicos (Histosoles, $133.7 \mathrm{~kg} \mathrm{~m}^{-2}$ ); esto se debe a los valores bajos de $\mathrm{pH}$, estado nutrimental y condiciones anaeróbicas que contribuyen a la acumulación del carbono (Tarnocai, 1998).

En los suelos orgánicos de manglares, los promedios de descomposición pueden ser más bajos y por lo tanto el potencial de almacenamiento de $\mathrm{C}$ del suelo puede ser más alto que en sistemas de agua dulce (Izaurralde et al., 2001). Los resultados de algunos estudios indican que la composición de los residuos de plantas, así como el incremento de la salinidad afecta el flujo superficial del $\mathrm{CO}_{2}$ y $\mathrm{C}$ orgánico disuelto 
durante la descomposición del residuo de plantas, pudiendo ser una consideración importante para el almacenamiento de dióxido de carbono $\left(\mathrm{CO}_{2}\right)$ en sistemas salinos, por lo tanto se considera a las plantas halófitas como una alternativa para la captura de C (Olsen et al., 1996). Además, Karmakar (1982) menciona que los residuos del mangle son el principal componente orgánico y su acumulación se atribuye a la intensa actividad biológica que acompaña su descomposición, en el período en que el suelo permanece con bajo contenido de agua.

Los factores ecológicos que se presentan en el estado de Tabasco han determinado el establecimiento de un mosaico diverso de vegetación, entre los que destacan los humedales. Estos se ubican en grandes extensiones planas distribuidas en forma paralela al cordón litoral, específicamente en la confluencia de los Ríos Grijalva-Usumacinta, en el extremo septentrional de la cuenca del Río Tonalá, en áreas aisladas entre los Ríos Puxcatán, Tacotalpa, Puyacatengo, Teapa, Pichucalco y Tinco (López, 1995).

Palma y Cisneros (2000) indican que en Tabasco, la mayor extensión está ocupada por pastizales (37.2\%), por vegetación hidrófila y manglares (29.17\%), y por selva alta perennifolia (3\%); dada la extensión que ocupan en el estado, es importante estudiar y conocer las reservas de carbono contenidas en estos ecosistemas.

\section{OBJETIVOS}

Cuantificar la reserva de carbono, caracterizar física y químicamente y clasificar taxonómicamente a los suelos orgánicos con vegetación natural de mangle, de la parte oeste de Tabasco, México.

\section{METODOLOGÍA}

El área de estudio se localiza en la parte oeste del estado de Tabasco, México, en el límite con Veracruz y comprende parte de los municipios de Huimanguillo y Cárdenas (Fig. 1). El clima es Am (f), cálido húmedo con abundantes lluvias en verano (de 1715 a $2159 \mathrm{~mm}$ ) y una temperatura media anual de $26.9{ }^{\circ} \mathrm{C}$ (INEGI, 2002); su hidrología esta comprendida por el sistema del Río Tonalá, que incluye lagunas y pantanos de agua dulce y salobre, los cuales ocupan la mayor parte de la zona y se caracterizan por vegetación hidrófita, (West et al., 1987); su geología está conformada por sedimentos de materiales orgánicos recientes sobre antiguos sedimentos palustres, ambos del periodo Cuaternario (West et al., 1985); los suelos son clasificados como Histosoles por Palma y Cisneros (1998).

Los manglares en Tabasco se encuentran distribuidos en la parte posterior del cordón litoral, bordeando las lagunas que se extienden paralelas a la costa y que tienen conexión con el mar; su presencia está condicionada a las situaciones de inundación y concentración de sales disueltas en agua; las especies dominantes en la región son: mangle rojo (Rhizophora mangle L.), mangle blanco (Laguncularia racemosa (L.) Gaertn) y mangle prieto (Avicennia germinans (L.) L.) (García y Palma, 1998).

\section{Trabajo de campo}

Para la selección de los sitios de muestreo se realizó un reconocimiento de la zona a través de recorridos de campo, con base en los trabajos de Palma et al. (1998) y Moreno (1999). Se eligieron dos sitios representativos de mangle: uno rojo y otro blanco (Fig. 1).

Cuando el muestreo se realizó (diciembre de 2000), el nivel de agua se presentó a los $30 \mathrm{~cm}$ (mangle rojo) y 10 $\mathrm{cm}$ (mangle blanco) de profundidad, por lo que las muestras de suelo se colectaron a través de barrenas y cavahoyos; primero, se identificaron los diferentes horizontes o capas y profundidades de los materiales orgánicos por los diferentes grados de 


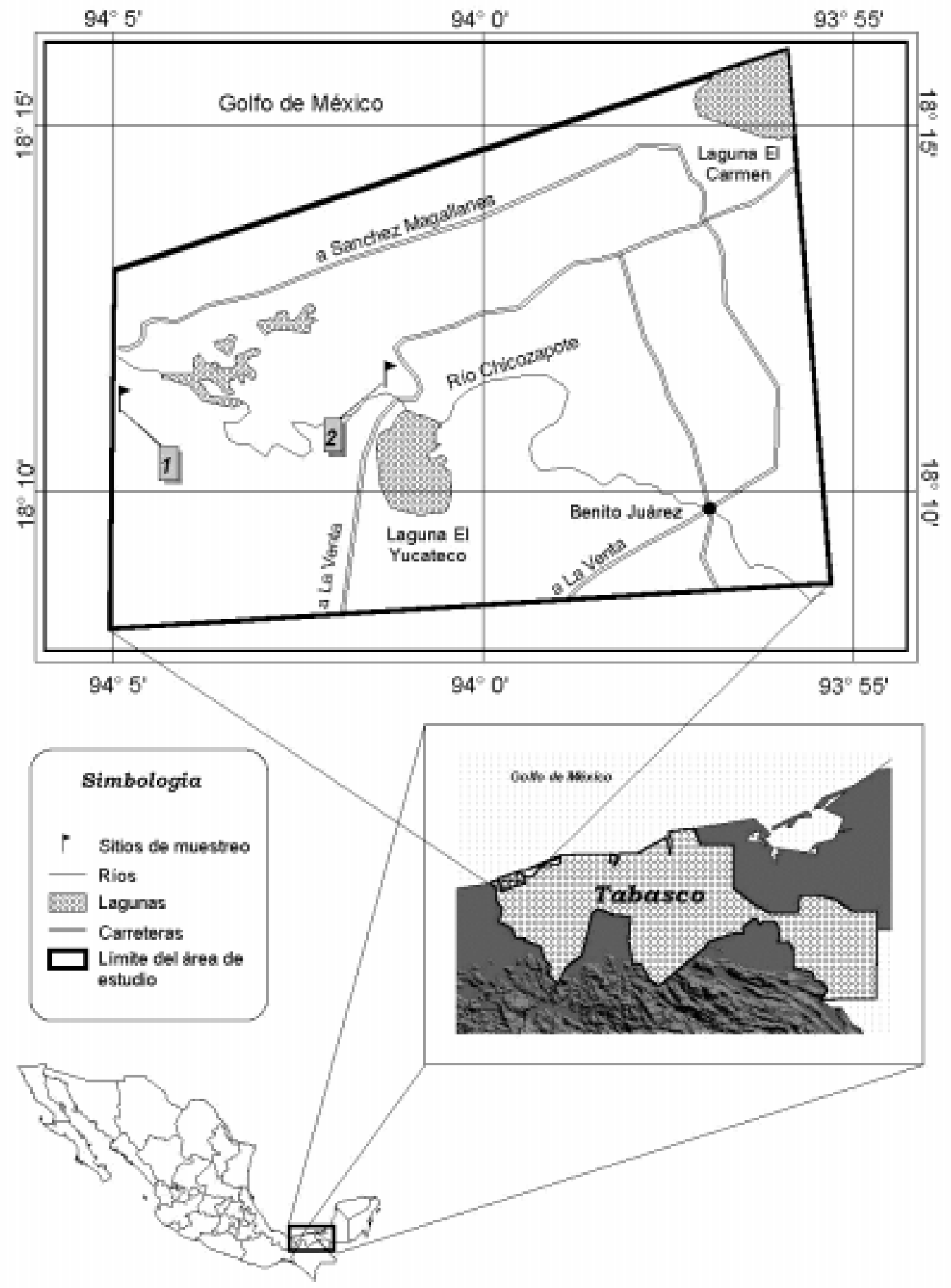

Figura 1. Localización del área de estudio y sitios de muestreo Fuente: INEGI, 1996; Moreno, 1999. Proyección: Geográfica. Datum:WGS 84 
descomposición (Soil Survey Staff, 1999) y posteriormente se realizó el muestreo; $140 \mathrm{~cm}$ (mangle rojo) y $200 \mathrm{~cm}$ (mangle blanco) de profundidad. Las muestras fueron llevadas al laboratorio para su secado a la sombra y a temperatura ambiente. Las propiedades del suelo que se analizaron fueron: $\mathrm{pH}$ (con muestra húmeda en $\mathrm{CaCl}_{2}$ y con muestra seca en $\mathrm{H}_{2} \mathrm{O}$, a una relación 1:4); conductividad eléctrica (CE) en pasta de saturación; capacidad de intercambio catiónico (CIC), con acetato de amonio $1 \mathrm{~N} \mathrm{pH} \mathrm{7;} \mathrm{sulfato}$ $\left(\mathrm{SO}_{4}\right)$ soluble en agua; nitrógeno total $\left(\mathrm{N}_{\mathrm{t}}\right)$, por el método micro-Kjeldahl. Todos los métodos usados están descritos en Van Reeuwijk (ISRIC, 1995).

Para determinar la densidad aparente (DA) fue necesario colectar nuevamente muestras de suelo inalteradas; para ello se esperó que el nivel del agua bajara de 20 $\mathrm{cm}$ a $25 \mathrm{~cm}$ de profundidad en los humedales, lo cual ocurrió en abril de 2001. Se utilizó el método del cilindro de volumen conocido y se realizó el muestreo sólo en la capa superficial.

La cuantificación de la materia orgánica y de carbono orgánico (CO) se realizó en muestras secadas a temperatura ambiente, y con un tamaño de partícula $<2 \mathrm{~mm}$, es decir, sin considerar las fibras mayores a este tamaño. Se utilizó el método de combustión seca, el cual consiste en la oxidación de la materia orgánica por calentamiento a $375^{\circ} \mathrm{C}$, y su posterior estimación por gravimetría (Kalra y Maynard, 1991). Este método se recomienda en suelos con altos contenidos de materia orgánica.

\section{RESULTADOS}

\section{Características morfológicas, físicas y químicas de los suelos}

Los dos suelos analizados con mangle rojo y mangle blanco se clasificaron como Typic Sulfosaprist, que significa que son Histosoles formados por materiales orgánicos en etapa avanzada de descomposición (horizontes genéticos designados como $\mathrm{Oa}$ ) y con materiales sulfhídricos (Fig. 2 y 3 ).

Sin embargo, de acuerdo con el arreglo y grado de descomposición de los materiales orgánicos, el perfil de suelo con mangle rojo, está compuesto principalmente por material sáprico; mientras que, en el perfil del mangle blanco, los dos primeros horizontes corresponden a material hémico y los tres siguientes a material sáprico. Sáprico significa que el material orgánico está en la etapa más avanzada de descomposición, Fíbrico, cuando está poco des-compuesto y aún se reconoce el material vegetal original y Hémico, es un estado de descomposición intermedia entre los anteriores (Soil Survey Staff, 1999).

Debido a que las muestras de suelo orgánico fueron tamizadas para separar las partículas $<2 \mathrm{~mm}$, tal como lo establece el método, gran parte de las fibras del mangle de la superficie quedaron sobre el tamiz, de ahí que no se clasificaran como horizontes orgánicos fíbricos.

Se ha descrito que los Histosoles de Tabasco, son suelos que pasan la mayor parte del año inundados y sólo durante 30 a 50 días, el agua desciende, permitiendo la aireación del horizonte superficial, lo que no sucede en los horizontes más profundos (Palma y Cisneros, 2000). El agua es el principal componente en suelos de humedales y las condiciones temporales ó permanentes de anaerobiosis son las que determinan la mayoría de los procesos que en ellos se desarrollan (Brady y Weil, 1999).

La descomposición microbial es más rápida en presencia de oxígeno, el cual actúa como receptor de electrones durante la oxidación aeróbica de compuestos orgánicos, pero el oxígeno disminuye cuando los poros están llenos de agua, entonces funcionan los organismos anaerobios o facultativos y la descomposición se vuelve lenta acumulándose grandes cantidades de materia 


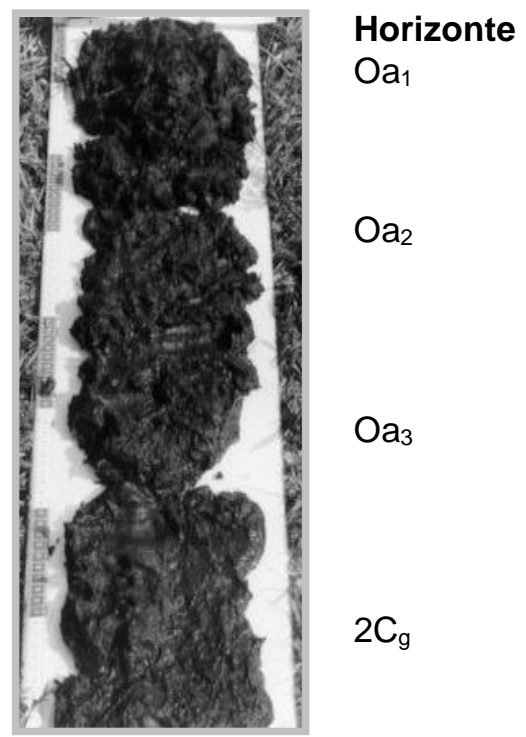

\section{Descripción}

0-30 cm; Capa con material fíbrico con muchas fibras; color pardo muy oscuro 10 YR 2/2; humedad: saturado; raíces abundantes y gruesas; $\mathrm{pH}$ : 6 en campo; pobremente drenado; transición a la siguiente capa: tenue e irregular.

30-60 cm; Capa con material orgánico ligeramente descompuesto; color pardo muy oscuro 10 YR 2/2; humedad: saturado; presencia de fibras y material ligeramente pegajoso; raíces comunes y medias; $\mathrm{pH}$ : 6.5 en campo; pobremente drenado; transición a la siguiente capa tenue e irregular.

60-90 cm; Capa con material orgánico muy descompuesto; color pardo muy oscuro 10 YR 2/2; humedad: saturado; material pegajoso; raíces: pocas y delgadas; $\mathrm{pH}$ : 6 en campo; pobremente drenado; transición a la siguiente capa: marcada e irregular.

90-140 cm; Capa con material arenoso; color pardo grisáceo muy oscuro 10 YR 3/2; humedad: saturado; textura de arena migajosa; raíces raras y finas; $\mathrm{pH}$ : 6 en campo; suelo pobremente drenado.

Figura 2. Descripción morfológica del perfil uno, manglar rojo (sulfosaprist).
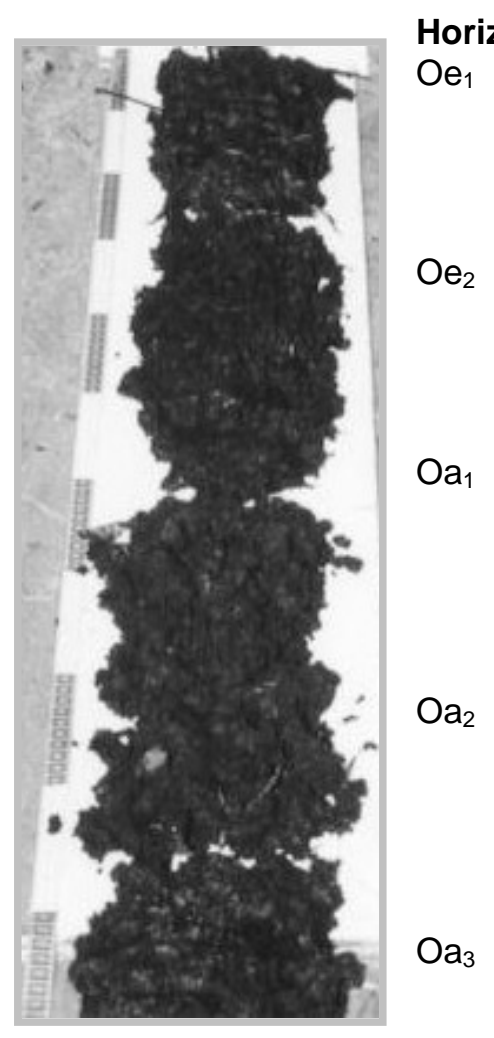

\section{Descripción}

0-30 cm; Capa con material fíbrico; color pardo muy oscuro 10 YR 2/2; humedad: saturado; consistencia: no pegajoso; raíces: abundantes y gruesas; $\mathrm{pH}$ : 7 en campo; pobremente drenado; transición a la siguiente capa: tenue.

30-60 cm; Capa con material orgánico fíbrico; color pardo muy oscuro 10 YR 2/2; humedad: saturado; consistencia: no pegajoso; raíces: comunes y medias; $\mathrm{pH}$ : 7 en campo; pobremente drenado; transición a la siguiente capa: tenue.

60-100 cm; Capa con material orgánico con mucha fibra; color pardo muy oscuro 10 YR 2/2; humedad: saturado; consistencia: no pegajoso; raíces: comunes y finas; $\mathrm{pH}$ : 7 en campo; pobremente drenado; transición a la siguiente capa: marcada.

100-140 cm; Capa con material orgánico muy descompuesto; color pardo muy oscuro 10 YR 2/2; humedad: saturado; consistencia: ligeramente pegajoso; raíces: pocas y finas; $\mathrm{pH}$ : 7 en campo; pobremente drenado; transición a la siguiente capa: marcada.

140-200 cm; Capa con material orgánico muy descompuesto; color negro 10 YR 2/1; humedad: saturado; consistencia: ligeramente pegajoso; raíces: muy pocas y finas; $\mathrm{pH}$ : 7.5 en campo; suelo pobremente drenado.

Figura 3. Descripción morfológica del perfil dos, manglar blanco (sulfosaprist). 
orgánica (Brady y Weil, 1999), tal como sucede en los suelos de manglar que fueron estudiados.

Yefremova (1993) menciona que la dominancia de materiales sápricos, se debea la descomposición de los restos vegetales durante el tiempo que baja el nivel del agua y se dispone de oxígeno. En el caso de estos suelos, se mantienen condiciones de anaerobiosis durante la mayor parte del año, por lo que se esperaría que no hubiese descomposición de la materia orgánica; sin embargo, la temperatura del área está favoreciendo su degradación al bajar la lámina de agua.

Los suelos estudiados tienen una densidad aparente (DA) de $0.22 \mathrm{~g} \mathrm{~cm}^{-3} \mathrm{y}$ $0.09 \mathrm{~g} \mathrm{~cm}^{-3}$ en el primer y segundo sitio, respectivamente. Estas densidades aparentes bajas están relacionadas con el tipo de material orgánico, contenido mineral y humedad durante el muestreo (Lucas, 1982; Skopp, 2000) y corroboran la presencia de materiales sápricos y hémicos.

En relación con las propiedades químicas, en el sitio 1 , el $\mathrm{pH}$ en $\mathrm{CaCl}_{2}$ varía de neutro a moderadamente ácido y con agua de fuertemente a extremadamente ácido; en el sitio 2 , el $\mathrm{pH}$ en $\mathrm{CaCl}_{2}$ es de neutro a ligeramente ácido, mientras que en $\mathrm{H}_{2} \mathrm{O}$ es fuertemente ácido (Tablas 1 y 2), según la clasificación de Aguirre (2001). Estos pH fuertemente ácidos están relacionados con el suplemento de sulfato (agua de mar) y materia orgánica (mangle); el sulfato y el oxígeno son adicionados por el movimiento de la marea a través de la alta permeabilidad de los suelos de mangle (Breemen y Buurman, 1998). Batlle-Sales et al., (1994) señalan que la reducción de sulfatos del agua de mar acumulados en el suelo, genera la formación de materiales sulfihídricos y la exposición a condiciones de secado acelera la oxidación bioquímica de los materiales orgánicos y de los sulfihídricos, de ahí que disminuya drásticamente el $\mathrm{pH}$ y que se clasifiquen como Sulfosaprist.
La conductividad eléctrica (CE) para ambos sitios es superior a los $4 \mathrm{dS} \mathrm{m}^{-1}$ en todos sus horizontes, por lo que se consideran como suelos salinos (Richards, 1954); lo anterior se atribuye a la influencia del agua marina que los inunda por períodos prolongados (Tablas 1 y 2$)$.

En cuanto a los contenidos de materia orgánica (MO), son altos en los dos sitios (hasta $48.3 \%$, en mangle rojo y hasta $85.7 \%$, en el mangle blanco), lo cual es común en suelos que permanecen inundados durante mucho tiempo y con aportes constantes de materia orgánica (Kilham y Alexander, 1984). El sitio 2 tuvo mayor contenido de materia orgánica debido a la fisiografía del área, ya que por estar mas alejado del cordón litoral tiene menor contacto mineral y esto lo convierte en un suelo orgánico más profundo (Tablas 1 y 2 ).

La CIC resultó también muy alta en todos los horizontes orgánicos a pesar del $\mathrm{pH}$ fuertemente ácido. La materia orgánica tiene grupos funcionales principalmente carboxílicos y fenólicos con cargas que varían según el $\mathrm{pH}$ del sistema, es decir, negativas en $\mathrm{pH}$ alcalinos y positivas en pH ácidos (Cottenie, 1980). El pH también contribuye a que la $\mathrm{CIC}$ sea alta ya que a valores altos un mayor número de hidrógenos son liberados de los ácidos débiles (Baize, 1993; Tan, 1998).

Los suelos presentan un alto contenido de $\mathrm{Nt}$, con valores mayores o iguales a 0.4 , siendo mayor en el segundo sitio; además, presentan una alta relación $\mathrm{C}: \mathrm{N}$ que refleja la dificultad que tienen los microorganismos para degradar los residuos vegetales (Tablas 1 y 2 ).

\section{Acumulación de carbón orgánico (CO) en suelos de manglar}

Con los datos de carbón orgánico (CO), se hizo la estimación de su posible reserva en $\mathrm{Mg} \mathrm{ha}^{-1} \mathrm{o}$ en $\mathrm{kg} \mathrm{m}^{-2}$, con el objeto de comparar la acumulación de la 
Tabla 1. Caracterización química de un Histosol con mangle rojo (Rhizophora mangle)

(Sitio 1) en el oeste de Tabasco, México

\begin{tabular}{|c|c|c|c|c|c|c|c|c|c|}
\hline \multirow{2}{*}{$\begin{array}{l}\text { PROF. } \\
(\mathrm{cm})\end{array}$} & \multirow{2}{*}{ HORIZONTE } & \multicolumn{2}{|c|}{$\mathrm{pH}$} & $\mathrm{MO}$ & $\mathrm{N}_{\mathrm{t}}$ & \multirow[t]{2}{*}{ C:N } & \multirow{2}{*}{$\begin{array}{c}\mathrm{ClC} \\
\left(\mathrm{cmol} \mathrm{kg}^{-1}\right)\end{array}$} & \multirow{2}{*}{$\begin{array}{c}\mathrm{CE} \\
\left(\mathrm{dS} \mathrm{m}^{-1}\right)\end{array}$} & \multirow{2}{*}{$\begin{array}{l}\mathrm{SO}_{4} \\
(\%)\end{array}$} \\
\hline & & $\mathrm{CaCl}_{2}$ & $\mathrm{H}_{2} \mathrm{O}(1: 4)$ & \multicolumn{2}{|c|}{$(\%)$} & & & & \\
\hline $0-30$ & $\mathrm{Oa}_{1}$ & 5.6 & 4.5 & 48.3 & 0.5 & 59.8 & 43.7 & 43.6 & 0.3 \\
\hline $30-60$ & $\mathrm{Oa}_{2}$ & 6.5 & 4.7 & 35.8 & 0.4 & 52.5 & 43.2 & 55.0 & 0.3 \\
\hline $60-90$ & $\mathrm{Oa}_{3}$ & 6.0 & 4.8 & 38.7 & 0.4 & 56.7 & 40.6 & 54.3 & 0.3 \\
\hline $90-140$ & $2 \mathrm{C}_{\mathrm{g}}$ & 6.0 & 2.8 & 6.9 & 0.1 & 40.2 & 8.5 & 53.0 & 0.9 \\
\hline
\end{tabular}

Tabla 2. Caracterización química de un Histosol con mangle blanco (Laguncularia racemosa) (Sitio 2) en el oeste de Tabasco, México

\begin{tabular}{|c|c|c|c|c|c|c|c|c|c|}
\hline \multirow{2}{*}{$\begin{array}{l}\text { PROF. } \\
\text { (cm) }\end{array}$} & \multirow[t]{2}{*}{ HORIZONTE } & \multicolumn{2}{|r|}{$\mathrm{pH}$} & MO & $\mathrm{N}_{\mathrm{t}}$ & \multirow[t]{2}{*}{$\mathrm{C}: \mathrm{N}$} & \multirow{2}{*}{$\begin{array}{c}\text { CIC } \\
\mathrm{cmol} \mathrm{kg}^{-1}\end{array}$} & \multirow{2}{*}{$\begin{array}{c}C E \\
\left(d S ~ m^{-1}\right)\end{array}$} & \multirow{2}{*}{$\begin{array}{l}\mathrm{SO}_{4} \\
(\%)\end{array}$} \\
\hline & & $\mathrm{CaCl}_{2}$ & $\mathrm{H}_{2} \mathrm{O}(1: 4)$ & \multicolumn{2}{|c|}{ (\%) } & & & & \\
\hline $0-30$ & $\mathrm{Oe}_{1}$ & 5.6 & 4.7 & 85.7 & 2.1 & 23.4 & 134.4 & 12.1 & 0.3 \\
\hline $30-60$ & $\mathrm{Oe}_{2}$ & 6.3 & 4.6 & 85.8 & 2.0 & 24.6 & 130.4 & 35.4 & 0.3 \\
\hline $60-100$ & $\mathrm{Oa}_{1}$ & 6.2 & 5.2 & 83.6 & 1.7 & 27.8 & 70.0 & 47.2 & 0.2 \\
\hline $100-140$ & $\mathrm{Oa}_{2}$ & 6.4 & 5.5 & 75.9 & 1.3 & 34.4 & 104.0 & 85.5 & 0.4 \\
\hline $140-200$ & $\mathrm{Oa}_{3}$ & 6.0 & 4.8 & 70.4 & 1.1 & 39.1 & 100.7 & 112.3 & 0.6 \\
\hline
\end{tabular}

materia orgánica con estudios realizados en otras regiones. Esta estimación se obtuvo de la siguiente manera:

$$
\begin{aligned}
& \text { Mg Cha-1 o kg C m } \mathrm{m}^{-2}= \\
& \text { [peso del suelo] [\% CO] }
\end{aligned}
$$

donde:

Peso del suelo $\left[\right.$ t ha $\left.^{-1}\right]=$ [profundidad $][\mathrm{DA}]$

El sitio 1 tuvo un contenido total de $47.1 \mathrm{~kg} \mathrm{C} \mathrm{m}^{-2}$, esta acumulación tiene un grosor aproximado de $90 \mathrm{~cm}$ de profundidad (Fig. 4a); el sitio 2 tuvo un contenido mayor, esto es $82.2 \mathrm{~kg} \mathrm{C} \mathrm{m}^{-2}$ con un grosor de aproximadamente $200 \mathrm{~cm}$ de profundidad (Fig. 4b).
Por otro lado, el contenido total de C en $\mathrm{Mg} \mathrm{ha}^{-1}$ del sitio 1 fue 471.9 y en el segundo 822; estos valores son el resultado de un proceso de incorporación y acumulación de restos vegetales que han requerido miles de años (Tablas 3 y 4).

La estimación de la reserva de carbono en los manglares es complicada por la carencia de datos de densidad aparente. Eswaran et al. (1993) señalan que se debe a las condiciones permanentes de inundación lo cual dificulta la obtención de las muestras. En el caso de los suelos estudiados, estos permanecen inundados casi todo el año, aun en los meses de menor precipitación, por lo que fue necesario esperar a que el nivel de agua bajara y se pudieran colectar las muestras. 
A

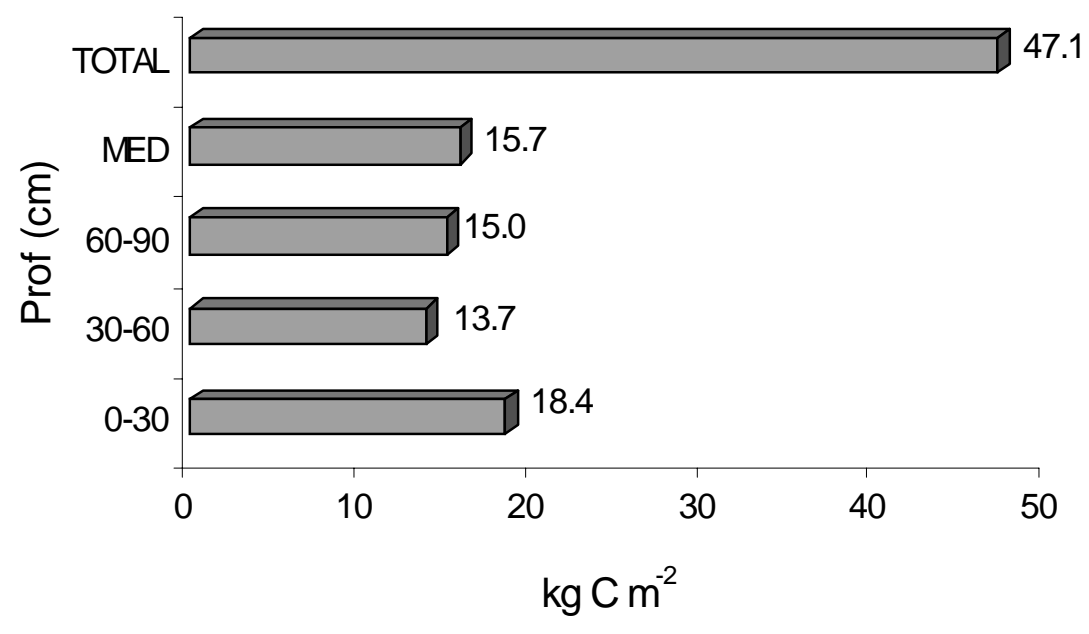

B

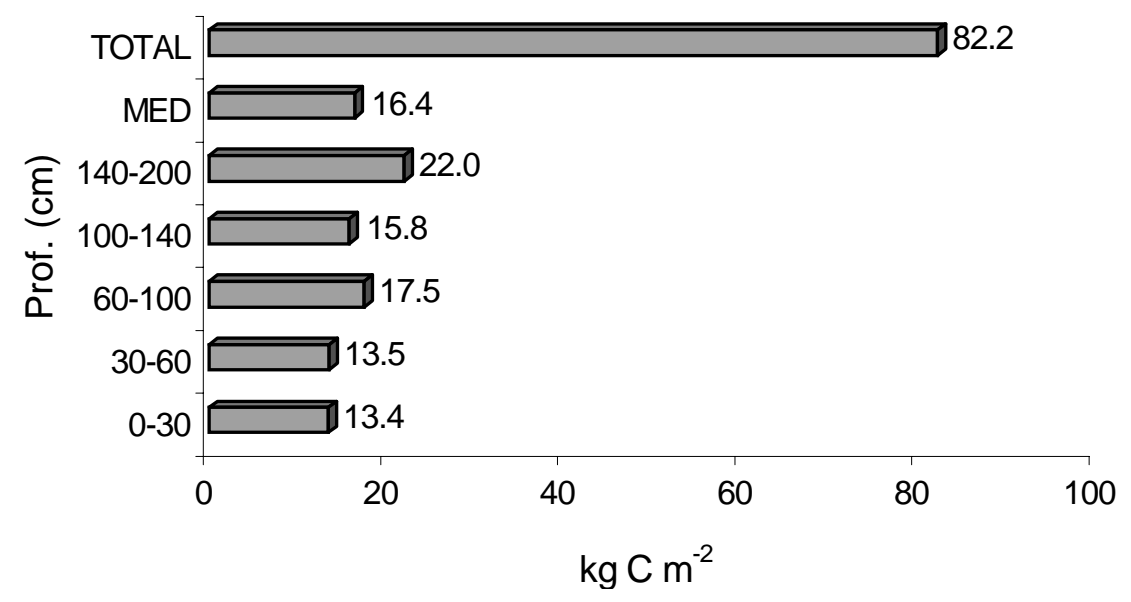

Figura 4. Distribución de $\mathrm{C}$ en el perfil de suelo en manglares del oeste de Tabasco (A: sitio 1, B: sitio 2).

Tabla 3. Reserva natural de $\mathrm{C}$ en Histosol con mangle rojo (Rhizophora mangle) ( sitio 1), en el oeste de

Tabasco, México

\begin{tabular}{ccccc}
\hline PROF. & HORIZONTE & $\begin{array}{c}\text { CO } \\
\%\end{array}$ & FACTOR & $\mathrm{Mg} \mathrm{C} \mathrm{ha}^{-1}$ \\
\hline $0-30$ & $\mathrm{Oa}_{1}$ & 27.9 & 660 & 184.14 \\
\hline $30-60$ & $\mathrm{Oa}_{2}$ & 20.8 & 660 & 137.28 \\
\hline $60-90$ & $\mathrm{Oa}_{3}$ & 22.8 & 660 & 150.48 \\
\hline $90-140$ & $2 \mathrm{C}_{\mathrm{g}}$ & 4.1 & $*$ & $*$ \\
\hline TOTAL & & & & 471.9 \\
\hline MEDIA & & & & 157.3 \\
\hline
\end{tabular}

Factor: peso de suelo $\left(\mathrm{t} \mathrm{ha}^{-1}\right)$ y DA 
Tabla 4. Reserva natural de $\mathrm{C}$ en un Histosol con mangle blanco (Laguncularia racemosa) (sitio 2), en el oeste de Tabasco, México

\begin{tabular}{ccccc}
\hline PROF. & HORIZONTE & $\begin{array}{c}\text { CO } \\
\%\end{array}$ & FACTOR & $\mathrm{Mg} \mathrm{C} \mathrm{ha}^{-1}$ \\
\hline $0-30$ & $\mathrm{Oe}_{1}$ & 49.7 & 270 & 134.19 \\
\hline $30-60$ & $\mathrm{Oe}_{2}$ & 49.8 & 270 & 134.46 \\
\hline $60-100$ & $\mathrm{Oa}_{1}$ & 48.5 & 360 & 174.6 \\
\hline $100-140$ & $\mathrm{Oa}_{2}$ & 44 & 360 & 158.4 \\
\hline $140-200$ & $\mathrm{Oa}_{3}$ & 40.8 & 540 & 220.3 \\
\hline TOTAL & & & & 822.0 \\
\hline MEDIA & & & & 164.4 \\
\hline
\end{tabular}

Factor: peso de suelo (t ha ${ }^{-1}$ ) y DA

La acumulación de carbono orgánico en humedales es primeramente un resultado del balance de dos procesos: fijación de carbono a través de la fotosíntesis y pérdida por la descomposición; los promedios de fotosíntesis son más altos que en otros ecosistemas y el promedio de descomposición es más lento debido a las condiciones anaeróbicas, por lo que la materia orgánica tiende a acumularse (Reddy et al., 2000).

La acumulación y descomposición de la materia orgánica en los manglares indica su desarrollo, además, está relacionado con su posición en el paisaje y con el tipo de vegetación. En el primer sitio, el espesor orgánico tiene $90 \mathrm{~cm}$ y sobreyace a sedimentos fluviomarinos (horizonte 2C $\mathrm{C}_{\mathrm{g}}$ ) (López, 1995); la acumulación de la materia orgánica no es tan intensa debido a que los manglares están ubicados en el cordón litoral. En el sitio 2, los horizontes orgánicos alcanzan más de $200 \mathrm{~cm}$ de profundidad debido a que no se llegó hasta el horizonte $2 \mathrm{C}_{\mathrm{g}}$, además, hay una mayor diferenciación de horizontes, por los diferentes grados de descomposición. Este suelo se localiza en un ambiente más estable que ha permitido la acumulación gradual de la materia orgánica. Lucas (1982), menciona que la acumulación de un metro de "peat" (material fíbrico originado principalmente por vegetación de musgo) en condiciones frías, requiere en promedio de 1500 años para su formación. Lo que puede significar que la formación de estos suelos orgánicos, menos fibrosos que el peat, requirió de varios miles de años para su formación.

En cuanto al tipo de vegetación, la acumulación de hojarasca sobre el piso del manglar puede ser un importante factor para la inmovilización de nutrientes durante la descomposición; en bosques de Rhizophora (mangle rojo), la tasa de descomposición de hojarasca es más baja y la inmovilización de nitrógeno es más alta, que en bosques de Avicennia (mangle blanco), como resultado de una alta tasa C:N (Yáñez et al., 1998). De acuerdo con este comportamiento se esperaría mayor acumulación y horizontes fíbricos en el mangle rojo pero su ubicación en el paisaje lo limita. En el sitio 2, la estabilidad del paisaje y la alta tasa de descomposición ha permitido la acumulación de materiales sápricos, sin 
embargo, este ecosistema es muy frágil en el sentido de que se pueden alterar y degradar fácilmente, si por ejemplo se exponen a la superficie.

Comparando los resultados indicados en la Tabla 4 con los de la Tabla 5, se observa que los suelos orgánicos formados bajo comunidades de mangle, tienen un gran potencial como reserva de C y pueden desempeñar un papel importante en la regulación de la concentración de $\mathrm{CO}_{2}$ atmosférico (Zhong y Qiguo, 2001).

El conocimiento sobre contenido de $\mathrm{C}$, puede contribuir a las estimaciones recientes y futuras de la captura de este elemento por los ecosistemas tropicales; sobre todo si se considera que cuando estos suelos son drenados o expuestos a condiciones aeróbicas, pueden llegar a oxidarse y en el proceso liberar grandes cantidades de carbono a la atmósfera.

\section{CONCLUSIONES}

Este estudio arroja un primer estimado del contenido de carbono orgánico en Histosoles con vegetación de mangle en el oeste de Tabasco, México. La cantidad que se registró es mayor a lo presentado en investigaciones realizadas en otras regiones con diferentes usos del suelo. Sin embargo, es necesario llevar a cabo mediciones de biomasa del mangle en el área de estudio, así como determinaciones de carbono en la vegetación, para estimar el secuestro del carbono por parte de este ecosistema.

Tabla 5. Cuadro comparativo de almacenaje de carbono orgánico realizado en diferentes lugares y usos de suelo

\begin{tabular}{lcc}
\hline SITIO O USO DEL SUELO & $\begin{array}{c}\text { ALMACENAJE DE } \\
\text { CARBONO } \\
\left(\mathrm{kg} \mathrm{C} \mathrm{m} \mathrm{m}^{-2}\right.\end{array}$ & REFERENCIA \\
\hline Montaña Olímpica, EUA & $3.5-14.5$ & Pichard et al., 2000 \\
\hline China Tropical y subtropical & & Zhong y Qiguo, 2001 \\
Pantanos de vegetación herbácea & 40.0 & \\
Suelos bajo coníferas & 19.6 & \\
Bosques de hoja ancha & 19.2 & \\
Suelos de arrozales & 12.6 & \\
Tierras altas & 9.4 & \\
Pastos de sabana & 10.5 & \\
\hline Brasil & & \\
Bosques vírgenes & $13.0-37.0$ & \\
Selva secundaria & $10.0-20.0$ & \\
Suelos de sábana & $9.0-16.0$ & \\
Oxisol & $12.0-24.0$ & Rabenhorst y \\
\hline Suelo agrícola & $2.0-10.0$ & Swanson, 2000 \\
Histosol & $9.0-191.0$ & \\
\hline
\end{tabular}




\section{REFERENCIAS}

Aguirre, G. A. 2001. Química de los suelos ácidos, templados y tropicales. 1 a . ed. Universidad Nacional Autónoma de México. México, D. F. 289 p.

Baize, D. 1993. Soil science analyses, a guide to current use. Ed. John Wiley \& Sons. Nueva York, EUA.

Batlle S., J.; A. Abad F.; V. Bordás y E. Pepiol. 1994. Soil transformations in salt-stressed lagoon ecosystems. In: J.D. Etchevers B. ed. Memorias del $X V$ Congreso Internacional de la Ciencia del Suelo. Acapulco, México. p:262-269 .

Brady, N.C. y R.R. Weil. 1999. The nature and properties of soils. 9 ${ }^{\underline{a}}$ ed. Prentice-Hall. EUA. 881 p.

Breemen, N.V. y P. Buurman. 1998. Soil formation. Kluwer Academic Publishers. Holanda. 377 p.

Cottenie, A. 1980. Los análisis de suelos y plantas como base para formular recomendaciones sobre fertilizantes. Boletín de suelos de la FAO 38/2. FAO, Roma, Italia.

Eswaran, H.; E. Van Den Berg y P. Reich 1993. Organic carbon in soils of the world. Soil Sci. Soc. Am. J. 57:192194.

Food and Agriculture Organization of the United Nations (FAO). 1989. Carta mondiale des sols legende revisée. Rapport sur les ressources en sols du monde. No. 60. FAO/UNESCO. Roma, Italia. $125 \mathrm{p}$.

García, L.E. y D.J. Palma L. 1998. Asociación suelo-vegetación en tres zonas fisiográficas de Tabasco. In: $\mathrm{P}$. Magaña, ed. Libro de resúmenes del VII Congreso Latinoamericano de Botánica y XIV Congreso Mexicano de Botánica. Universidad Autónoma Metropolitana. México, D.F. p:68.

Izaurralde, R.C.; N.J. Rosenberg y R. Lal. 2001. Mitigation of climatic change by soil carbon sequestration: issues of science, monitoring, and degraded lands. Advances in Agronomy. 70:175.

Karmarkar, S.M. 1982. Senescence in mangroves. In: W. Junk, ed. Contributions to the ecology of halophytes. La Haya, Holanda. p: 173.

Kilham, O.W. y M. Alexander. 1984. A basis for organic matter accumulation in soils under anaerobiosis. Soil Science. 137 (6):419-427.

Lal, R. 2001. World cropland soils as a source or sink for atmospheric carbon. Advances in Agronomy. 71:145-191.

Lal, R., J. Kimble y R. Follet. 1998. Pedospheric processes and the carbon cycle. In: Lal, R.; J.M. Kimble; R.F. Follet y B.A. Stewart, eds. Soil Processes and the carbon cycle. Ohio. p:1-8.

López, M. 1995. Tipos de vegetación y su distribución en el Estado de Tabasco y Norte de Chiapas. 1 $1^{\underline{a}}$ edición. Universidad Autónoma Chapingo. Chapingo, México.

Lucas, R.E. 1982. Organic soils (Histosols) formation, distribution, physical and chemical properties and management for crop production. Research report. The Michigan State University.

Neill, Ch.; C. Cerri; J.M. Melillo; B.J. Feigl; P.A. Steudler; J.F.L. Moraes y M.C. Piccolo. 1998. Stocks and dynamics of soils Carbon following deforestation for pasture in rondonia. 
In: Lal, R.; J.M. Kimble; R.F. Follet y B.A. Stewart, eds. Soil Processes and the carbon cycle. Ohio. p: 9-28.

Olsen, M.W.; R.J. Frye y E.P. Glenn. 1996. Effect of salinity and plant species on $\mathrm{CO}_{2}$ flux and leaching of dissolved organic carbon during decomposition of plant residue. Plant and soil. 179:183-188.

Palma L., D.J. y J. Cisneros D. 2000. Plan de uso sustentable de los suelos de Tabasco. $2^{\underline{a}}$ ed. ISPROTABFundación Produce Tabasco-Colegio de Post-graduados. Villahermosa, Tabasco. México. 118 p.

Prichard, S.J.; D.L. Peterson y R.D. Hammer. 2000. Carbon distribution in subalpine Forests and Meadows of the Olimpic Mountains, Washington. Soil Sci. Am. J. 64:1834-1845.

Rabenhorst M.C. y D. Swanson. 2000. Clasification of soils: Histosols. E183:E-208 p. In: Sumner M.E., ed. Handbook of soil science. CRC PRESS. Nueva York, EUA.

Reddy K.R.; E.M. D'Angelo y W.G. Harris. 2000. Biogeochemistry of wetlands. G-89:G114 p. In: Sumner M.E., ed. Handbook of soil science. CRC PRESS. Nueva York, EUA.

Richards, L.A., ed. 1954. Diagnosis and improvement of saline and alkali soils. USDA, Agriculture Handbook. No. 60. Washington, D.C.

Skoop, J.M. 2000. Physical properties of primary particles. In: Sumner M. E. ed. Handbook of soil science. CRC PRESS. Nueva York, EUA. p:A-1-16.

Soil Survey Staff. 1999. Keys to Soil Taxonomy. Soil Conservation
Service. 2a. ed. United States Department of Agriculture. USDA. State University. Washington, D.C. EUA.

Tan, K. H. 1998. Principles of soil chemistry. $3^{\mathrm{a}}$ ed. Marcel Decker. Nueva York, EUA.

Tarnocai, D. 1998. The amount of organic carbon in various soil orders and ecological provinces in Canada In: Lal, R.; J.M. Kimble; R.F. Follet y B.A. Stewart, eds. Soil Processes and the carbon cycle. Ohio State University. EUA. p:81-92.

International Soil Reference and Information Centre (ISRIC). 1995. Procedimientos para análisis de suelos (clasificación y correlación). Van Reeuwijk, ed. Traducción al español. M.C. Gutiérrez C.; C.A. Tavaes E. y C.A. Ortiz S. Especialidad de Edafología. Colegio de Postgraduados. Montecillo, Texcoco, México. 145 p.

Wallace, D. 2000. Capture and storage of $\mathrm{CO}_{2}$, what needs to be done?. IEA Greenhouse Gases R \& D Programmer. París, Francia. 10 p.

Yáñez A., A., R.R. Twilley y A.L. Lara D. 1998. Los ecosistemas de manglar frente al cambio climático global. Madera y Bosques. 4(2):3-19.

Yefremova, T. G. 1993. Pedogenesis and diagnosis of peaty soils of marsh ecosystems. Eurasian Soil Science. 25 (5):28-37.

Zhong, L. y Z. Qiguo. 2001. Organic carbon content and distribution in soils under different land uses in tropical and subtropical China. Plant and Soil. 231:175-185.» 
1 Colegio de Postgraduados. Especialidad de Edafología. Área de génesis, morfología y clasificación de suelos. km 36.5 Carretera México-Texcoco, Montecillo 56180 Edo. de México. c.e.: ecaliz@ colpos.colpos.mx.

2 Colegio de Postgraduados-Campus Tabasco. Periférico Carlos A. Molina s/n. km 3.5 Apdo. Postal 24. Cárdenas 86500 Tabasco.

Manuscrito recibido el 6 de diciembre de 2001.

Aceptado el 12 de febrero de 2002.

Este documento se debe citar como:

Moreno C., E; A. Guerrero P.; M.C. Gutiérrez C.; C.A. Ortiz S. y D.J. Palma L. 2002. Los manglares de Tabasco, una reserva natural de carbono. Madera y Bosques Número especial:115-128. 